\title{
Variance of the quantum dwell time for a nonrelativistic particle
}

\author{
G. E. Hahne * \\ M. S. T27B-1, NASA/Ames Research Center \\ Moffett Field, California 94035 USA
}

January 19, 2012

PACS Numbers: 03.65.Ca, 03.65.Xp

\begin{abstract}
Muñoz, Seidel, and Muga [Phys. Rev. A 79, 012108 (2009)], following an earlier proposal by Pollak and Miller [Phys. Rev. Lett. 53, 115 (1984)] in the context of a theory of a collinear chemical reaction, showed that suitable moments of a two-flux correlation function could be manipulated to yield expressions for the mean quantum dwell time and mean square quantum dwell time for a structureless particle scattering from a timeindependent potential energy field between two parallel lines in a twodimensional spacetime. The present work proposes a generalization to a charged, nonrelativistic particle scattering from a transient, spatially confined electromagnetic vector potential in four-dimensional spacetime. The geometry of the spacetime domain is that of the slab between a pair of parallel planes, in particular those defined by constant values of the third $(z)$ spatial coordinate. The mean $N^{\text {th }}$ power, $N=1,2,3, \ldots$, of the quantum dwell time in the slab is given by an expression involving an $N$ flux-correlation function. All these means are shown to be nonnegative. The $N=1$ formula reduces to an $S$-matrix result published previously [G. E. Hahne, J. Phys. A 36, 7149 (2003)]; an explicit formula for $N=2$, and of the variance of the dwell time in terms of the $S$-matrix, is worked out. A formula representing an incommensurability principle between variances of the output-minus-input flux of a pair of dynamical variables (such as the particle's time flux and others) is derived.
\end{abstract}

\section{Introduction}

In 1984, Pollak and Miller [1] proposed a formula for computing the quantum mean time for the system of a forward or reverse collinear chemical reaction $\mathrm{AB}+\mathrm{C} \leftrightarrow \mathrm{A}+\mathrm{BC}$ to dwell between two spatial surfaces exterior to, and including, the reaction zone. Their formalism depended on calculating the algebraic

*Electronic address: Gerhard.E.Hahne@nasa.gov 
sum of weighted averages of the two-flux correlation function between the normal fluxes of particles at the same, or opposite, pairs of the spatial surfaces, and was based on a microcanonical ensemble for choosing the density matrix of the quantum mixture. In 2009, Muñoz, Seidel, and Muga ([2], Eq. (14)) reformulated the two-flux method of Pollak and Miller to allow the estimation of mean powers of the dwell time in potential scattering in one spatial dimension; they in effect presumed a density matrix derived from a pure quantum state. They showed that the two-flux formalism could be manipulated and reduced to a known single-flux method for computing the mean dwell time, and that the mean-square dwell time formula in the context of Pollak and Miller's approach was mathematically equivalent to a previously known, but distinct, formula also involving a two-flux correlation function.

Muñoz, et al. also advanced a formula for the mean-cube quantum dwell time in terms of Pollak and Miller's two-flux correlation functions, and gave or implied another formula for the mean $N^{\text {th }}$ power of the dwell time in terms of $N$-flux correlation functions. For $N=3$, in the free-particle case, the two formulas gave different results.

In the present work, a general formula for the mean $N^{\text {th }}$ power of the dwell time for a particle between a pair of $z=$ constant planes containing the interaction zone, in terms of $N$-flux correlation functions, is proposed. The physical system is that of a charged, structureless particle moving in four-dimensional spacetime, and scattering from a specified, transient, spatially confined, electromagnetic four-vector potential. The formulas for $N=1$ and $N=2$ reduce to those of [2], and the results for all higher powers reduce to a straightforward generalization of [2], rhs of Eq. (18). An incommensurability principle will be derived that establishes a lower bound to the product of variances of the output-minus-input fluxes of a pair of distinct dynamical variables of the particle.

The ensuing material is organized as follows: In Section 2, we shall define general $\mathrm{N}$-flux correlation functions for the probability four-currents at an ordered set of $N$ points, $N=1,2,3, \ldots$, of a simple charged particle moving in a time-dependent electromagnetic field. The correlation function will prove to have zero four-divergence with respect to each set of coordinates and associated vector indices (generalizing [2], Eq. (9)). We shall show further that a weighted average of such a correlation function of normally-directed currents on the direct product of $N$ three-dimensional spacetime boundaries equals, by an $N$-fold application of Gauss's theorem, to a $4 N$-dimensional volume integral that is a weighted sum of the current correlations in the direct product of the interior spacetime volumes. Section 3 applies the method to propose a general formula for the mean $N^{\text {th }}$ power of the dwell time; the formula entails the computation of the $N$-flux correlation function of the particle's probability current, and can be expressed either as an integral over the product of $N$ copies of a four-dimensional slab-like spacetime domain, or as integrals over the product of $N$ copies of the three-dimensional boundary of the domain. All mean powers of the dwell time calculated according to the general formula are shown to be nonnegative, thereby satisfying an important physical criterion. We then show that the general formula reduces to results given in [2] for the mean first and 
mean second powers of the dwell time. In Section 4, an explicit formula for the mean-square dwell time in terms of the $S$-matrix is derived, and it is shown the mean-square dwell time is greater than, or equal to, the square of the mean dwell time -hence, the variance of the dwell time is well defined. There are four appendixes: In Appendix A, the properties of the $U$ operators, which are expressed in the familiar form $\exp \left[-i\left(t_{b}-t_{a}\right) H / \hbar\right]$ for time-independent Hamiltonians, are studied particularly in the context of spacewise propagation of wave functions, and where the Hamiltonians are time-dependent. In Appendix B, a formula giving a lower bound to the product of the variances of two delocalized observables in terms of the mean value of a generalized commutator of the observables is derived. In Appendix C, an expression closely resembling the Aharonov-Bohm operator for arrival time is shown to arise from calculating the mean flux of time across a single $z=$ constant surface in spacetime. Appendix D gives arguments that are intended to provide insight into the presence of flux correlations in the computations involving delocalized (i.e., for measurements not confined to a single timelike or spacelike hyperplane) observables.

\section{Single-particle, multiple-point-current corre- lation functions}

In this section we shall define, and study some properties of $N^{\text {th }}$-order $(N=$ $1,2,3, \ldots)$ correlations of the probability flow currents of a simple charged particle moving in a given background electromagnetic field. The space of quantum states will be that of suitably well-behaved functions of the four real coordinates labeling points in spacetime:

$$
\xi=\left(\xi^{\mu}\right)=\left(\xi^{0}, \xi^{1}, \xi^{2}, \xi^{3}\right)=(t, x, y, z)=\left(t, r^{1}, r^{2}, r^{3}\right)=(t, \mathbf{r}) .
$$

Matrix elements of operators between quantum states in Dirac notation will entail integrals over a four-dimensional domain $D^{4}$ of spacetime:

$$
\langle\Phi|\Omega| \Psi\rangle_{D^{4}}=\int_{D^{4}} d^{4} \xi_{1} \int_{D^{4}} d^{4} \xi_{2} \Phi\left(\xi_{1}\right)^{*}\left\langle\xi_{1}|\Omega| \xi_{2}\right\rangle \Psi\left(\xi_{2}\right)
$$

where we omit $\mid \Omega$ on the lhs and substitute $\delta^{4}\left(\xi_{1}-\xi_{2}\right)$ for $\left\langle\xi_{1}|\Omega| \xi_{2}\right\rangle$ on the rhs if $\Omega$ is the unit operator on spacetime functions. It will normally be the case that $\Phi(\xi)$ and $\Psi(\xi)$ in the above are both solutions of the Schrödinger equation. Convergence problems can be dealt with by restricting the operators used, the spacetime domain involved, or the wave functions.

The dynamics is given by the nonrelativistic Schrödinger equation, and the components of the four-vector current operator have the usual form in terms of the derivative operators and the electromagnetic four-vector potential ([3], Eq. (24.39), and [4], Eqs. (11.16) and (11.22)). In fact, we have the specified four-vector electromagnetic-field potential

$$
\left(A^{\mu}(\xi)\right)=(V(\xi), \mathbf{A}(\xi))
$$


and the Schrödinger equation for $\Psi(\xi)$ of a particle with mass $m$ and charge $e$

$$
\begin{aligned}
i \hbar \frac{\partial}{\partial t} \Psi(\xi) & =\frac{1}{2 m} \sum_{j=1}^{3}\left[\frac{\hbar}{i} \frac{\partial}{\partial r^{j}}-\frac{e}{c} A^{j}(\xi)\right]\left[\frac{\hbar}{i} \frac{\partial}{\partial r^{j}}-\frac{e}{c} A^{j}(\xi)\right] \Psi(\xi) \\
& +e V(\xi) \Psi(\xi) .
\end{aligned}
$$

We define the spacetime matrix of the four-current operator $\mathcal{J}^{\mu}(\Xi)$ at position $(\Xi)=(T, \mathbf{R})$ as follows:

$$
\begin{aligned}
& \left\langle t_{2}, \mathbf{r}_{2}\left|\mathcal{J}^{\mu}(T, \mathbf{R})\right| t_{1} \cdot \mathbf{r}_{1}\right\rangle=\delta\left(t_{2}-T\right) \delta\left(T-t_{1}\right) \\
& \times \begin{cases}\delta^{3}\left(\mathbf{r}_{1}-\mathbf{R}\right) \delta^{3}\left(\mathbf{R}-\mathbf{r}_{2}\right) & \text { if } \mu=0 ; \\
\delta^{3}\left(\mathbf{r}_{2}-\mathbf{R}\right)\left[\frac{\hbar}{2 i m}\left(\underline{\frac{\partial}{\partial R^{j}}}-\frac{\partial}{\partial R^{j}}\right)\right. & \\
\left.\quad-\frac{e}{m c} A^{j}(T, \mathbf{R})\right] \delta^{3}\left(\mathbf{R}-\mathbf{r}_{1}\right), & \text { if } \mu=j=1,2,3,\end{cases}
\end{aligned}
$$

where the under-arrows indicate the direction in which the derivative acts when the operator is sandwiched into a matrix element. Note that the matrices of the $\mathcal{J}^{\mu}(\Xi)$ operators are all Hermitean, at least on the space of differentiable, complex-valued test functions on spacetime. One can also verify that, if both $\Phi(\xi)$ and $\Psi(\xi)$ are solutions of (4), then the four-divergence of the associated (and in general, complex-valued) current distribution is everywhere zero (the summation convention on a $\mu=0,1,2,3$ is operative henceforth):

$$
\frac{\partial}{\partial \Xi^{\mu}}\left\langle\Phi\left|\mathcal{J}^{\mu}(\Xi)\right| \Psi\right\rangle=0
$$

Now let $\Psi(\xi ; \mathbf{k})$, where $\mathbf{k}$ ranges over all real three-vectors, be a complete, orthonormal set of solutions to (4), in that at every time $t$,

$$
\int d^{3} k \Psi\left(t, \mathbf{r}_{2} ; \mathbf{k}\right) \Psi\left(t, \mathbf{r}_{1} ; \mathbf{k}\right)^{*}=\delta^{3}\left(\mathbf{r}_{2}-\mathbf{r}_{1}\right) ;
$$

orthonormality will be defined in terms of the matrix $\mathcal{F}^{0}(T)$ in a wave vector basis,

$$
\begin{aligned}
\left\langle\Psi\left(\mathbf{k}_{2}\right)\right| & \mathcal{F}^{0}(T)\left|\Psi\left(\mathbf{k}_{1}\right)\right\rangle \\
& =\int d^{3} R\left\langle\Psi\left(\mathbf{k}_{2}\right)\left|\mathcal{J}^{0}(T, \mathbf{R})\right| \Psi\left(\mathbf{k}_{1}\right)\right\rangle \\
& =\delta^{3}\left(\mathbf{k}_{2}-\mathbf{k}_{1}\right) .
\end{aligned}
$$

An analogous flux-related matrix, called $\mathcal{F}^{3}\left(R^{3}\right)$ for $z$-propagation, will be defined in (A11).

The vector $\mathbf{k}$ will be taken to be the wave vector of an input plane wave such that the state $\Psi(t, \mathbf{r} ; \mathbf{k})$ contains purely outgoing scattered waves. We can now define a $U^{(1)}$ operator that propagates solutions of (4) in time, where the superscript (1) is intended to indicate that not free-particle states, but 
interacting-particle states are involved; the symbol $U^{(0)}$ will be used for the corresponding operator in the interaction-free case - see (A7ab). We take

$$
\left\langle t_{2}, \mathbf{r}_{2}\left|U^{(1)}\right| t_{1}, \mathbf{r}_{1}\right\rangle=\int d^{3} k \Psi\left(t_{2}, \mathbf{r}_{2} ; \mathbf{k}\right) \Psi\left(t_{1}, \mathbf{r}_{1} ; \mathbf{k}\right)^{*},
$$

which by (7) reduces to the unit operator on a time $=$ constant slice when $t_{1}=t_{2}$. Note that the operator $U^{(1)}$ yields a Hermitean matrix in spacetime, in that

$$
\left\langle t_{2}, \mathbf{r}_{2}\left|U^{(1)}\right| t_{1}, \mathbf{r}_{1}\right\rangle^{*}=\left\langle t_{1}, \mathbf{r}_{1}\left|U^{(1)}\right| t_{2}, \mathbf{r}_{2}\right\rangle .
$$

We shall also make use of a generalized density operator which has a complete matrix in spacetime. Let $\tilde{\rho}\left(\mathbf{k}_{2} ; \mathbf{k}_{1}\right)$ be a Hermitean, positive semidefinite matrix with unit trace that represents the particular mixture of plane-wave states that existed at times before the particle interacts significantly with the electromagnetic field. The spacetime matrix of the density operator $\rho^{(1)}$ is taken to be

$$
\left\langle t_{2}, \mathbf{r}_{2}\left|\rho^{(1)}\right| t_{1}, \mathbf{r}_{1}\right\rangle=\int d^{3} k_{1} \int d^{3} k_{2} \Psi\left(t_{2}, \mathbf{r}_{2} ; \mathbf{k}_{2}\right) \tilde{\rho}\left(\mathbf{k}_{2} ; \mathbf{k}_{1}\right) \Psi\left(t_{1}, \mathbf{r}_{1} ; \mathbf{k}_{1}\right)^{*} .
$$

The matrix of both operators $U^{(1)}$ and $\rho^{(1)}$ evidently satisfy (4) on the left spacetime arguments, and the complex-conjugate equation applied to the right spacetime arguments.

We now define a quantum average of an ordered $N$-point current correlation function:

$$
\begin{aligned}
\left(J^{\mu_{1} \mu_{2} \ldots \mu_{N}}\right. & \left.\left(\Xi_{1} ; \Xi_{2} ; \ldots ; \Xi_{N}\right)\right)_{\tilde{\rho}} \\
& =\operatorname{Tr}\left\langle\rho^{(1)} \mathcal{J}^{\mu_{1}}\left(\Xi_{1}\right) U^{(1)} \mathcal{J}^{\mu_{2}}\left(\Xi_{2}\right) U^{(1)} \ldots \mathcal{J}^{\mu_{N}}\left(\Xi_{N}\right)\right\rangle .
\end{aligned}
$$

In view of (9) and (11), (12) reduces to

$$
\begin{aligned}
\left(J^{\mu_{1} \ldots \mu_{N}}\right. & \left.\left(\Xi_{1} ; \ldots ; \Xi_{N}\right)\right)_{\tilde{\rho}}=\int d^{3} k_{1} \ldots \int d^{3} k_{N+1} \tilde{\rho}\left(\mathbf{k}_{N+1}, \mathbf{k}_{1}\right) \\
& \times\left\langle\Psi\left(\mathbf{k}_{1}\right)\left|\mathcal{J}^{\mu_{1}}\left(\Xi_{1}\right)\right| \Psi\left(\mathbf{k}_{2}\right)\right\rangle \cdots\left\langle\Psi\left(\mathbf{k}_{N}\right)\left|\mathcal{J}^{\mu_{N}}\left(\Xi_{N}\right)\right| \Psi\left(\mathbf{k}_{N+1}\right)\right\rangle .
\end{aligned}
$$

Since in (13) each $\mathcal{J}^{\mu}(\Xi)$ is sandwiched between two solutions of (4), (6) implies that the four-divergence of the lhs of (12) or (13) at each of the $N$ points is zero:

$$
\frac{\partial}{\partial \Xi^{\mu_{s}}}\left(J^{\mu_{1} \ldots \mu_{N}}\left(\Xi_{1} ; \ldots ; \Xi_{N}\right)\right)_{\tilde{\rho}}=0, \text { for each } s=1,2, \ldots, N .
$$

We suppose that the particle is effectively free on or near the boundary of, and everywhere outside, the slab $S^{4}$ in spacetime, defined as follows:

$$
S^{4}=\left\{(T, \mathbf{R}) \mid-\infty<T, R^{1}, R^{2}<\infty, z_{a}<R^{3}<z_{b}\right\} .
$$

The boundary $\partial S^{4}$ of the slab comprises the two three-dimensional planes defined by

$$
\partial S^{4}=\left\{(T, \mathbf{R}) \mid-\infty<T, R^{1}, R^{2}<\infty, R^{3}=z_{a} \text { or } R^{3}=z_{b}\right\} .
$$


We define the weighted current matrix as follows. Let $w_{s}\left(\xi_{s}\right), s=1,2, \ldots, N$, be a sequence of suitable real-valued weight functions. Then we take

$$
\begin{aligned}
\left\langle\xi_{s}\left|W^{\mu_{s}}\left(\Xi_{s}\right)\right| \xi_{s+1}\right\rangle & =(1 / 2)\left[w_{s}\left(\xi_{s}\right)\left\langle\xi_{s}\left|\mathcal{J}^{\mu_{s}}\left(\Xi_{s}\right)\right| \xi_{s+1}\right\rangle\right. \\
& \left.+\left\langle\xi_{s}\left|\mathcal{J}^{\mu_{s}}\left(\Xi_{s}\right)\right| \xi_{s+1}\right\rangle w_{s}\left(\xi_{s+1}\right)\right]
\end{aligned}
$$

where the symmetrization of the operator $\mathcal{J}$ with the weight function $w$ is done because for $\mu=1,2,3$, the current operator includes differentiations so generally doesn't commute with $w$. We can use the delta-functions to replace the arguments of $w$ by $\Xi$; because of the particular bidirectionality of the differentiation process the terms involving differentiation of $w$ cancel out, so that we can factor the $w$ out of the quantum matrix element:

$$
\left\langle\Psi\left(\mathbf{k}_{s}\right)\left|W^{\mu_{s}}\left(\Xi_{s}\right)\right| \Psi\left(\mathbf{k}_{s+1}\right)\right\rangle=\left\langle\Psi\left(\mathbf{k}_{s}\right)\left|\mathcal{J}^{\mu_{s}}\left(\Xi_{s}\right)\right| \Psi\left(\mathbf{k}_{s+1}\right)\right\rangle w_{s}\left(\Xi_{s}\right) .
$$

Because of (6), the four-divergence of such a matrix is

$$
\frac{\partial}{\partial \Xi_{s}^{\mu_{s}}}\left\langle\Psi\left(\mathbf{k}_{s}\right)\left|W^{\mu_{s}}\left(\Xi_{s}\right)\right| \Psi\left(\mathbf{k}_{s+1}\right)\right\rangle=\left\langle\Psi\left(\mathbf{k}_{s}\right)\left|\mathcal{J}^{\mu_{s}}\left(\Xi_{s}\right)\right| \Psi\left(\mathbf{k}_{s+1}\right\rangle \frac{\partial w_{s}}{\partial \Xi_{s}^{\mu_{s}}} .\right.
$$

The weighted mean $N$-order current is now well-defined as follows:

$$
W_{\tilde{\rho}}^{\mu_{1} \ldots \mu_{N}}\left(\Xi_{1} ; \ldots ; \Xi_{N}\right)=\left(J^{\mu_{1} \ldots \mu_{N}}\left(\Xi_{1} ; \ldots ; \Xi_{N}\right)\right)_{\tilde{\rho}} \prod_{s=1}^{N} w_{s}\left(\Xi_{s}\right) .
$$

We further define the mean total divergence (creation) $\bar{W}_{\tilde{\rho}}$ of the quantity $\prod_{s=1}^{N} w_{s}$ in the slab as the $N$-fold integral over the slab of the $N$-fold divergence of the current density $W$ :

$$
\bar{W}_{\tilde{\rho}}=\int_{S^{4}} d^{4} \Xi_{1} \ldots \int_{S^{4}} d^{4} \Xi_{N} \frac{\partial^{N}}{\partial \Xi_{1}^{\mu_{1}} \ldots \partial \Xi_{N}^{\mu_{N}}} W_{\tilde{\rho}}^{\mu_{1} \ldots \mu_{N}}\left(\Xi_{1} ; \ldots ; \Xi_{N}\right) .
$$

In view of (19), we have

$$
\bar{W}_{\tilde{\rho}}=\int_{S^{4}} d^{4} \Xi_{1} \ldots \int_{S^{4}} d^{4} \Xi_{N}\left(J^{\mu_{1} \ldots \mu_{N}}\left(\Xi_{1} ; \ldots ; \Xi_{N}\right)\right)_{\tilde{\rho}} \prod_{s=1}^{N} \frac{\partial w_{s}}{\partial \Xi_{s}^{\mu_{s}}} .
$$

An $N$-fold application of Gauss's theorem to (21) yields the following surface integral for $\bar{W}_{\tilde{\rho}}$ :

$$
\begin{aligned}
\bar{W}_{\tilde{\rho}} & =\int_{\partial S^{4}} d T_{1} d R_{1}^{1} d R_{1}^{2} \ldots \int_{\partial S^{4}} d T_{N} d R_{N}^{1} d R_{N}^{2} \\
& \times\left.\left. W_{\tilde{\rho}}^{3_{1} \ldots 3_{N}}\left(T_{1}, \mathbf{R}_{1} ; \ldots ; T_{N}, \mathbf{R}_{N}\right)\right|_{R_{1}^{3}=z_{a}} ^{R_{1}^{3}=z_{b}} \ldots\right|_{R_{N}^{3}=z_{a}} ^{R_{N}^{3}=z_{b}}
\end{aligned}
$$

The latter is a weighted average over $N$-flux correlation functions, generalizing the two-flux weighted averages used in [1] and [2]. 
We remark that a sum of product weight functions will also lead to a sum of entities as in (21) and (22). The particular choices of weight functions for $N=2$, that is, $-(1 / 2)\left|T_{1}-T_{2}\right|$ and $-(1 / 2)\left(T_{1}-T_{2}\right)^{2}$, provide alternative means of deriving [2], Eqs. (17) and (18), respectively, when expressed in the form of [5], Eq. (D2); note, however, that the first of these does not fit the pattern of a sum of products of functions of $T_{1}$ and $T_{2}$.

\section{Mean powers of the dwell time}

The methods of Sec. 2 permit the construction of expressions that are candidates for the expectation values of the $N^{\text {th }}$ powers of the dwell time- see also the discussion in Sec. 5. We take the weight functions $w_{s}$ to be

$$
w_{s}\left(\Xi_{s}\right)=\Xi_{s}^{0_{s}}=T_{s}, \text { for } s=1, \ldots, N,
$$

and factor these into (23), while calling the result $T_{S^{4} * * N}$. We have from (22)

$$
T_{S^{4} * * N}=\int_{S^{4}} d^{4} \Xi_{1} \cdots \int_{S^{4}} d^{4} \Xi_{N}\left(J^{0_{1} \ldots 0_{N}}\left(\Xi_{1} ; \ldots ; \Xi_{N}\right)\right)_{\tilde{\rho}} .
$$

In view of the simple structure of the operator $J^{0}(\Xi)$ (see $(5),(25)$ ),

$$
\begin{aligned}
\int_{S^{4}} d^{4} \Xi\left\langle\Psi(\mathbf{k})\left|J^{0}(\Xi)\right| \Psi\left(\mathbf{k}^{\prime}\right)\right\rangle_{S^{4}} & =\int_{S^{4}} d^{4} \xi \Psi(\xi ; \mathbf{k})^{*} \Psi\left(\xi ; \mathbf{k}^{\prime}\right) \\
& =\left\langle\Psi(\mathbf{k}) \mid \Psi\left(\mathbf{k}^{\prime}\right)\right\rangle_{S^{4}} \\
& =\left(\left\langle\Psi\left(\mathbf{k}^{\prime}\right) \mid \Psi(\mathbf{k})\right\rangle_{S^{4}}\right)^{*} .
\end{aligned}
$$

We now have

$$
\begin{aligned}
T_{S^{4} * * N} & =\int d^{3} k_{1} \cdots \int d^{3} k_{N+1} \tilde{\rho}\left(\mathbf{k}_{N+1} ; \mathbf{k}_{1}\right) \\
& \times\left\langle\Psi\left(\mathbf{k}_{1}\right) \mid \Psi\left(\mathbf{k}_{2}\right)\right\rangle_{S^{4}} \cdots\left\langle\Psi\left(\mathbf{k}_{N}\right) \mid \Psi\left(\mathbf{k}_{N+1}\right)\right\rangle_{S^{4}}
\end{aligned}
$$

The cases $N=1$ and $N=2$ are given or derived in [2], Eqs. (1) and (18), respectively. For larger $N,(27)$ reduces to, in the notation of [2], an expectation value of the operator $\left(\hat{T}_{D}\right)^{N}$.

We shall now show that the rhs of (27) is real and nonnegative for each $N \geq 1$. The proofs for even and odd $N$ differ slightly. For $N=2 M=2,4,6, \ldots$, we can rearrange the rhs of (27), as given by (13):

$$
\begin{aligned}
T_{S^{4} * * 2 M}= & \int d^{3} k_{M+1}\left[\int d^{3} k_{1} \cdots \int d^{3} k_{M} \int d^{3} k_{M+2} \cdots \int d^{3} k_{2 M+1}\right. \\
& \times\left(\left\langle\Psi\left(\mathbf{k}_{M+1}\right) \mid \Psi\left(\mathbf{k}_{M+2}\right)\right\rangle_{S^{4}} \cdots\left\langle\Psi\left(\mathbf{k}_{2 M}\right) \mid \Psi\left(\mathbf{k}_{2 M+1}\right)\right\rangle_{S^{4}}\right) \\
& \left.\times \tilde{\rho}\left(\mathbf{k}_{2 M+1}, \mathbf{k}_{1}\right)\left(\left\langle\Psi\left(\mathbf{k}_{1}\right) \mid \Psi\left(\mathbf{k}_{2}\right)\right\rangle_{S^{4}} \cdots\left\langle\Psi\left(\mathbf{k}_{M}\right) \mid \Psi\left(\mathbf{k}_{M+1}\right)\right\rangle_{S^{4}}\right)\right] .
\end{aligned}
$$


For each $\mathbf{k}_{M+1}$ the inner integral on the rhs of (26) is the expection value of a state (within large parentheses) with respect to the positive semidefinite density matrix $\tilde{\rho}$; the result $T_{S^{4} * * 2 M}$ is therefore real and nonnegative.

For $N=2 M-1=1,3,5, \ldots$, using (3), we rearrange as follows:

$$
\begin{aligned}
T_{S^{4} * * 2 M-1} & =\int_{S^{4}} d^{4} \xi_{M}\left[\int d^{3} k_{1} \cdots \int d^{3} k_{2 M}\right. \\
& \times\left(\Psi\left(\xi_{M} ; \mathbf{k}_{M+1}\right)^{*}\left\langle\Psi\left(\mathbf{k}_{M+1}\right) \mid \Psi\left(\mathbf{k}_{M+2}\right)\right\rangle_{S^{4}} \cdots\right. \\
& \left.\times\left\langle\Psi\left(\mathbf{k}_{2 M-1}\right) \mid \Psi\left(\mathbf{k}_{2 M}\right)\right\rangle_{S^{4}}\right) \\
& \times \tilde{\rho}\left(\mathbf{k}_{2 M} ; \mathbf{k}_{1}\right)\left(\left\langle\Psi\left(\mathbf{k}_{1}\right) \mid \Psi\left(\mathbf{k}_{2}\right)\right\rangle_{S^{4}} \cdots\right. \\
& \left.\left.\times\left\langle\Psi\left(\mathbf{k}_{M-1}\right) \mid \Psi\left(\mathbf{k}_{M}\right)\right\rangle_{S^{4}} \Psi\left(\xi_{M} ; \mathbf{k}_{M}\right)\right)\right]
\end{aligned}
$$

For each fixed $\xi_{M}$, the inner integral above is an expectation value with respect to $\tilde{\rho}$; hence $T_{S^{4} * * 2 M-1}$ is real and nonnegative.

Classically, a particle's trajectory can be contrived to be tangential to the boundary $\partial S^{4}$, whence its mean dwell time and all powers would be zero; for the present system, we shall avoid tangentially-moving wave function components $\left(k^{3}=0\right)$ due to mathematical singularities there. Nevertheless, one could choose a $\tilde{\rho}$ such that the particle moves fast enough normal to the slab that it spends an arbitrarily small, but necessarily nonzero, quantum time in $S^{4}$; zero is therefore the greatest possible lower bound for the quantum dwell time for a slab of any thickness.

\section{Variance of the dwell time}

In order for there to exist incommensurability relations for the dwell time of a particle in $S^{4}$ and other dynamical variables, it is necessary at least that there exist a nonnegative variance $V(T)_{S^{4}}$ of the dwell time, which should be given in terms of the mean square and mean dwell times as defined in (25), as follows:

$$
V(T)_{S^{4}}=T_{S^{4} * 2}-\left(T_{S^{4} * 11}\right)^{2} .
$$

The standard deviation $\Delta(T)$ of the dwell time for the domain $S^{4}$ will then be

$$
\Delta(T)_{S^{4}}=\left[V(T)_{S^{4}}\right]^{1 / 2} .
$$

We shall define presently an expression for the variance of the dwell time in $S^{4}$ that has the desired properties. The question of incommensurability of measuring the dwell time jointly with other dynamical variables is discussed in Appendix B. 
First, note that the result is trivial for the domain $Q^{4}$ defined below in (D1), since

$$
\begin{aligned}
T_{Q^{4} * * N} & =\int_{\partial Q^{4}} d^{3} R_{1} \cdots \int_{\partial Q^{4}} d^{3} R_{N} \\
& \times\left.\left.\left[T_{1} \cdots T_{N}\left(J^{0_{1} \ldots 0_{N}}\left(\Xi_{1} ; \ldots ; \Xi_{N}\right)\right)_{\tilde{\rho}}\right]\right|_{T_{1}=t_{a}} ^{T_{1}=t_{b}} \cdots\right|_{T_{N}=t_{a}} ^{T_{N}=t_{b}} \\
& =\left(t_{b}-t_{a}\right)^{N}, \text { for } N=1,2, \ldots
\end{aligned}
$$

The variance $V(T)_{Q^{4}}$ is therefore zero. This simple result carries over only partly into the predicted averages of powers of $\left(z_{b}-z_{a}\right)$ ("normal travel distance") of the end points of the slab $S^{4}$. In particular, if there is total reflection of the particle approaching either boundary, the normal travel distance of the particle inside the slab is zero, while if there is transmission but never a reflected signal, the normal travel distance is $\left(z_{b}-z_{a}\right)$, with a continuum of possible outcomes for partial transmission and reflection; a more complicated result obtains for the mean of the square of the normal travel distance [6].

We now define the variance of the dwell time so that it is nonnegative and satisfies (30):

$$
\begin{aligned}
V(T)_{S^{4}}= & \int d^{3} k_{1} \int d^{3} k_{2} \int d^{3} k_{3} \\
\times & {\left[\int_{S^{4}} d^{4} \Xi_{2} \frac{\partial}{\partial \Xi^{\mu_{2}}}\left\langle\Psi\left(\mathbf{k}_{3}\right)\left|\Xi_{2}^{0} \mathcal{J}^{\mu_{2}}\left(\Xi_{2}\right)\right| \Psi\left(\mathbf{k}_{2}\right)\right\rangle-T_{S^{4} * * 1} \delta^{3}\left(\mathbf{k}_{3}-\mathbf{k}_{2}\right)\right] } \\
& \times \tilde{\rho}\left(\mathbf{k}_{2} ; \mathbf{k}_{1}\right) \\
& \times\left[\int_{S^{4}} d^{4} \Xi_{1} \frac{\partial}{\partial \Xi^{\mu_{1}}}\left\langle\Psi\left(\mathbf{k}_{1}\right)\left|\Xi_{1}^{0} \mathcal{J}^{\mu_{1}}\left(\Xi_{1}\right)\right| \Psi\left(\mathbf{k}_{3}\right)\right\rangle-\delta^{3}\left(\mathbf{k}_{1}-\mathbf{k}_{3}\right) T_{S^{4} * * 1}\right] .
\end{aligned}
$$

The variance so defined is nonnegative, as for each fixed $\mathbf{k}_{3}$, the remaining expression is an expectation value of a state-vector-like entity with respect to the positive semidefinite $\tilde{\rho}$, and multiplying out the integrand yields four terms that reduce to $(30)$.

Next we shall give the formulas for $T_{S^{4} * * 1}$ and $T_{S^{4} * * 2}$ in terms of the $S$ matrix. The former was obtained in [7], Eqs. (89)-(92) (the closed-channel terms of [7], Eq. (93) play no role here); inasmuch as [7] used the wave vector comprising $\left(k^{t}, k^{x}, k^{y}\right)$ instead of the $\left(k^{x}, k^{y}, k^{z}\right)$ used herein, we shall state the old result in terms of the new variables. It is convenient first to introduce a Hermitean operator-function $\Lambda\left(z_{b}, z_{a}\right)$, which acts on suitable complex-valued 
functions in wave-vector space, that appears in both formulas:

$$
\begin{aligned}
\left\langle\Psi\left(\mathbf{k}_{5}\right)\right| & \Lambda\left(z_{b}, z_{a}\right)\left|\Psi\left(\mathbf{k}_{4}\right)\right\rangle=\left.\int_{\partial S^{4}} d T_{3} d R_{3}^{1} d R_{3}^{2}\left\langle\Psi\left(\mathbf{k}_{5}\right)\left|T_{3} \mathcal{J}^{3}\left(T_{3}, \mathbf{R}_{3}\right)\right| \Psi\left(\mathbf{k}_{4}\right)\right\rangle\right|_{R_{3}^{3}=z_{a}} ^{R_{3}^{3}=z_{b}} \\
= & \int d^{3} k_{6}\left[\delta ^ { 3 } ( \mathbf { k } _ { 5 } - \mathbf { k } _ { 6 } ) \left(-\frac{i}{2\left(k_{6}^{3}\right)^{2}}+\frac{i}{k_{6}^{3}} \frac{\partial}{\partial k_{6}^{3}}\right.\right. \\
& \left.+\frac{\theta\left(-k_{6}^{3}\right) z_{b}-\theta\left(k_{6}^{3}\right) z_{a}}{k_{2}^{3}}\right) \delta^{3}\left(\mathbf{k}_{6}-\mathbf{k}_{4}\right) \\
+ & S^{\dagger}\left(\mathbf{k}_{5} ; \mathbf{k}_{6}\right)\left(\frac{i}{2\left(k_{6}^{3}\right)^{2}}-\frac{i}{k_{6}^{3}} \frac{\partial}{\partial k_{6}^{3}}+\frac{\theta\left(k_{6}^{3}\right) z_{b}-\theta\left(-k_{6}^{3}\right) z_{a}}{k_{6}^{3}}\right) S\left(\mathbf{k}_{6} ; \mathbf{k}_{4}\right) \\
+ & \delta^{3}\left(\mathbf{k}_{5}-\mathbf{k}_{6}\right) \frac{i}{2 k_{6}^{3}\left|k_{6}^{3}\right|} \\
& \times\left(\theta\left(-k_{6}^{3}\right) \exp \left(-2 i k_{6}^{3} z_{b}\right)-\theta\left(k_{6}^{3}\right) \exp \left(-2 i k_{6}^{3} z_{a}\right)\right) S\left(k_{6}^{1}, k_{6}^{2},-k_{6}^{3} ; \mathbf{k}_{4}\right) \\
& +S^{\dagger}\left(\mathbf{k}_{5} ; k_{6}^{1}, k_{6}^{2},-k_{6}^{3}\right)\left(-\frac{i}{2 k_{6}^{3}\left|k_{6}^{3}\right|}\right) \\
& \left.\times\left(\theta\left(-k_{6}^{3}\right) \exp \left(2 i k_{6}^{3} z_{b}\right)-\theta\left(k_{6}^{3}\right) \exp \left(2 i k_{6}^{3} z_{a}\right)\right) \delta^{3}\left(\mathbf{k}_{6}-\mathbf{k}_{4}\right)\right]
\end{aligned}
$$

Note that the above is a differential operator that will act on arguments of the density matrix in the formulas to follow.

We remark that the terms linear in $S$ or $S^{\dagger}$ in (34) represent interference between forward- and backward-traveling waves at one or the other boundary plane. For an electron with energy $E=10^{-3} \mathrm{eV}$, the rationalized wave number $\left(2 m E / \hbar^{2}\right)^{1 / 2} \approx 2 \times 10^{8} \mathrm{~m}^{-1}$, so that the thickness $\left(z_{b}-z_{a}\right)$ of a slab would have to be roughly $10 \mathrm{~nm}$ or less in order that the exponents in these interference terms be small enough that oscillations - due to unavoidable slight smearing out of the energy distribution - do not average these terms to zero.

We remark also that in the free-particle case (unit $S$-matrix), the operator $\Lambda\left(z_{b}, z_{a}\right)$ reduces to (see also (C3))

$$
\begin{aligned}
\left\langle\Psi\left(\mathbf{k}_{5}\right)\right| & \left.\Lambda\left(z_{b}, z_{a}\right)\left|\Psi\left(\mathbf{k}_{4}\right)\right\rangle\right|^{\text {free }}=\delta^{3}\left(\mathbf{k}_{5}-\mathbf{k}_{4}\right)\left(\frac{z_{b}-z_{a}}{k_{4}^{3}}\right) \\
& +\delta^{1}\left(k_{5}^{1}-k_{4}^{1}\right) \delta^{1}\left(k_{5}^{2}-k_{4}^{2}\right) \delta^{1}\left(k_{5}^{3}+k_{4}^{3}\right) \\
& \times \frac{i}{2 k_{4}^{3}\left|k_{4}^{3}\right|}\left(\exp \left(2 i k_{4}^{3} z_{b}\right)-\exp \left(2 i k_{4}^{3} z_{a}\right)\right) .
\end{aligned}
$$

We can now state the formulas for the mean and mean square dwell time in $S^{4}$ :

$$
T_{S^{4} * * 1}=\frac{m}{\hbar} \int d^{3} k_{1} \int d^{3} k_{2}\left\langle\Psi\left(\mathbf{k}_{1}\right)\left|\Lambda\left(z_{b}, z_{a}\right)\right| \Psi\left(\mathbf{k}_{2}\right)\right\rangle \tilde{\rho}\left(\mathbf{k}_{2} ; \mathbf{k}_{1}\right),
$$

and

$$
\begin{aligned}
T_{S^{4} * * 2} & =\left(\frac{m}{\hbar}\right)^{2} \int d^{3} k_{1} \int d^{3} k_{2} \int d^{3} k_{3}\left\langle\Psi\left(\mathbf{k}_{3}\right)\left|\Lambda\left(z_{b}, z_{a}\right)\right| \Psi\left(\mathbf{k}_{1}\right)\right\rangle^{*} \\
& \times\left\langle\Psi\left(\mathbf{k}_{3}\right)\left|\Lambda\left(z_{b}, z_{a}\right)\right| \Psi\left(\mathbf{k}_{2}\right)\right\rangle \tilde{\rho}\left(\mathbf{k}_{2} ; \mathbf{k}_{1}\right) .
\end{aligned}
$$


In (37), the $\Lambda$-operators both differentiate only the density matrix, so their order is immaterial. It is worthy of note that the formula (37), and others (see App. B) herein, can involve the calculation of mean values of a vector-like entity, given the positive semidefinite density matrix $\tilde{\rho}$, where the "vector" can be a linear partial differential operator in $\mathbf{k}$-space; given suitable differentiability properties of $\tilde{\rho}\left(\mathbf{k}_{2} ; \mathbf{k}_{1}\right)$, it is plausible, but, so far as I can determine, not yet subject to mathematical investigation, whether the positive semidefinite property persists when the vector is of this type or possibly a generalized function. In the case of (36) and (37) the nonnegativity of these expressions was demonstrated in the mathematically distinct forms of (28) and (29), where the "vectors" are, or seem to be, ordinary functions.

\section{Appendix A: $U$ operators in spatial propagation}

In this Appendix we shall elaborate on the properties of plane-wave states, and on the free-particle $U^{(0)}$ and interacting particle $U^{(1)}$ operators in spatial propagation of quantum states.

First we consider the plane-wave states, which are solutions of the freeparticle Schrödinger equation.

$$
\psi(t, \mathbf{r} ; \mathbf{k})=(2 \pi)^{-3 / 2} \exp [i \mathbf{k} \cdot \mathbf{r}-i \hbar \mathbf{k} \cdot \mathbf{k} t /(2 m)] .
$$

These functions are orthonormal and complete on $t=$ constant slices of spacetime:

$$
\begin{aligned}
& \int d^{3} r \psi\left(t, \mathbf{r} ; \mathbf{k}_{1}\right)^{*} \psi\left(t, \mathbf{r} ; \mathbf{k}_{2}\right)=\delta^{3}\left(\mathbf{k}_{1}-\mathbf{k}_{2}\right), \\
& \int d^{3} k \psi\left(t, \mathbf{r}_{1} ; \mathbf{k}\right) \psi\left(t, \mathbf{r}_{2} ; \mathbf{k}\right)^{*}=\delta^{3}\left(\mathbf{r}_{1}-\mathbf{r}_{2}\right)
\end{aligned}
$$

The same plane waves are also orthogonal and qualifiedly normalized, but not complete, on $r^{3}=$ constant slices of spacetime, provided that we use the 3 component of the current operator instead of the unit operator as the entity with respect to which the matrix elements are calculated. We have

$$
\begin{aligned}
\int d t d r^{1} d r^{2} \psi & \left(t, r^{1}, r^{2}, r^{3} ; \mathbf{k}_{1}\right)^{*} \frac{\hbar}{2 i m}\left(\frac{\partial}{\partial r^{3}}-\frac{\partial}{\longleftrightarrow r^{3}}\right) \psi\left(t, r^{1}, r^{2}, r^{3} ; \mathbf{k}_{2}\right), \\
= & \frac{\hbar}{2 m}\left(k_{1}^{3}+k_{2}^{3}\right) \delta\left(k_{1}^{1}-k_{2}^{1}\right) \delta\left(k_{1}^{2}-k_{2}^{2}\right) \\
& \times \delta\left(\frac{\hbar}{2 m}\left(\left(k_{1}^{3}\right)^{2}-\left(k_{2}^{3}\right)^{2}\right)\right) \exp \left[i r^{3}\left(k_{2}^{3}-k_{1}^{3}\right)\right] \\
= & \sigma\left(k_{1}^{3}\right) \delta^{3}\left(\mathbf{k}_{1}-\mathbf{k}_{2}\right)
\end{aligned}
$$

where in the last step no contribution arises from the zero of the argument of the third $\delta$-function at $k_{2}^{3}=-k_{1}^{3}$, and

$$
\sigma\left(k^{3}\right)=k^{3} /\left|k^{3}\right| \text {. }
$$


As stated, we must avoid plane-wave input contributions from $k^{3} \approx 0$ in problems involving $S^{4}$. The plane wave states are not complete on the $r^{3}=$ constant surface, however, as only states of positive energy $\left(=\hbar k^{0}\right)$, which can be construed as the negative of the momentum conjugate to time, are involved. Since for "on shell" states

$$
k^{3}= \pm\left[\left(2 m k^{0} / \hbar\right)-\left(k^{1}\right)^{2}-\left(k^{2}\right)^{2}\right]^{1 / 2},
$$

those states for which the argument of the square root is negative have real exponential $r^{3}$-dependence, i.e., are closed channels. Closed-channel contributions appear in the Green's functions, but not in the $U^{(0)}$ operators-see [5], Eqs. (A17) and (A21).

The propagation operator $U^{(0)} \mathcal{J}^{3}$ gives rise to the following evolution of free-particle states in the $z$ direction:

$$
\begin{aligned}
\int d^{4} \xi_{2} \int d^{4} \xi_{3} \int & d T d R^{1} d R^{2}\left\langle\xi_{1}\left|U^{(0)}\right| \xi_{2}\right\rangle \\
\times & \left\langle\xi_{2}\left|\mathcal{J}^{3}\left(T, R^{1}, R^{2}, R^{3}\right)\right| \xi_{3}\right\rangle \psi\left(\xi_{3}\right) \\
& =\left(\Pi_{+} \psi\right)\left(\xi_{1}\right)-\left(\Pi_{-} \psi\right)\left(\xi_{1}\right),
\end{aligned}
$$

where $\psi$ is any solution of the free-particle Schrödinger equation having only real wave-vector (i.e., open-channel) components, and $\Pi_{+}$and $\Pi_{-}$are projection operators on the "positive $k^{3}$ " and "negative $k^{3}$ " parts of its operand-this result follows at once from (A3a). The minus sign for free-particle states with negative $k^{3}$ is desirable physically in that, for all terms in, say, $T_{S^{4} * * 1}$ or $T_{S^{4} * * 2}$, the particle passes the boundary planes in reverse order as those with positive $k^{3}$, and dwell times must be nonnegative. The calculation is more complicated in the interacting case as both reflections and transmissions can occur.

Let us next consider the operator $U^{(1)}$ in the temporal evolution $\left(Q^{4}\right)$ environment. With a plane wave (A1) as the input state, we infer from [7], following a translation of notation, that, given an input wave vector $\mathbf{k}_{1}$, the $S$-matrix plays the following role in the interacting-state wave functions of $(7),(8)$ :

$$
\begin{aligned}
& \Psi\left(t, \mathbf{r} ; \mathbf{k}_{1}\right)=\psi\left(t, \mathbf{r} ; \mathbf{k}_{1}\right), \text { for } t \leq t_{a}, \\
& \Psi\left(t, \mathbf{r} ; \mathbf{k}_{1}\right)=\int d^{3} k_{2} \psi\left(t, \mathbf{r} ; \mathbf{k}_{2}\right) S\left(\mathbf{k}_{2} ; \mathbf{k}_{1}\right), \text { for } t \geq t_{b} .
\end{aligned}
$$


We can now find explicit forms for $U^{(1)}$ outside or on $\partial Q^{4}$ :

$$
\begin{aligned}
& \left\langle t_{2}, \mathbf{r}_{2}\left|U^{(1)}\right| t_{1}, \mathbf{r}_{1}\right\rangle=\left\langle t_{2}, \mathbf{r}_{2}\left|U^{(0)}\right| t_{1}, \mathbf{r}_{1}\right\rangle=\int d^{3} k \psi\left(t_{2}, \mathbf{r}_{2} ; \mathbf{k}\right) \psi\left(t_{1}, \mathbf{r}_{1} ; \mathbf{k}\right)^{*}, \\
& \text { for } t_{2} \leq t_{a} \text { and } t_{1} \leq t_{a}, \text { or for } t_{2} \geq t_{b} \text { and } t_{1} \geq t_{b}, \\
& \left\langle t_{2}, \mathbf{r}_{2}\left|U^{(1)}\right| t_{1}, \mathbf{r}_{1}\right\rangle=\int d^{3} k_{2} \int d^{3} k_{1} \psi\left(t_{2}, \mathbf{r}_{2} ; \mathbf{k}_{2}\right) S\left(\mathbf{k}_{2} ; \mathbf{k}_{1}\right) \psi\left(t_{1}, \mathbf{r}_{1} ; \mathbf{k}_{1}\right)^{*}, \\
& \text { for } t_{2} \geq t_{b} \text { and } t_{1} \leq t_{a}, \\
& \left\langle t_{2}, \mathbf{r}_{2}\left|U^{(1)}\right| t_{1}, \mathbf{r}_{1}\right\rangle=\int d^{3} k_{2} \int d^{3} k_{1} \psi\left(t_{2}, \mathbf{r}_{2} ; \mathbf{k}_{2}\right) S^{\dagger}\left(\mathbf{k}_{2} ; \mathbf{k}_{1}\right) \psi\left(t_{1}, \mathbf{r}_{1} ; \mathbf{k}_{1}\right)^{*}, \\
& \text { for } t_{2} \leq t_{a} \text { and } t_{1} \geq t_{b},
\end{aligned}
$$

where we used the fact that $S$ is unitary.

Let $\mathbf{k}_{1}$ and $\mathbf{k}_{2}$ represent input and output wave vectors, respectively. The matrix $S\left(\mathbf{k}_{2} ; \mathbf{k}_{1}\right)$ comprises four blocks, according to the algebraic signs of its arguments $k_{2}^{3}$ and $k_{1}^{3}$, as follows:

$$
\begin{array}{ll}
k_{2}^{3}<0, k_{1}^{3}>0: & \text { output at } r^{3}=z_{a}, \text { input at } r^{3}=z_{a}, \text { (reflection), } \\
k_{2}^{3}>0, k_{1}^{3}<0: & \text { output at } r^{3}=z_{b}, \text { input at } r^{3}=z_{b},(\text { reflection}), \\
k_{2}^{3}>0, k_{1}^{3}>0: & \text { output at } r^{3}=z_{b}, \text { input at } r^{3}=z_{a}, \text { (transmission), } \\
k_{2}^{3}<0, k_{1}^{3}<0: & \text { output at } r^{3}=z_{a}, \text { input at } r^{3}=z_{b}, \text { (transmission). }
\end{array}
$$

(The above correspond to the open-channel reflection and transmission matrices of [7], Eqs. (73) and (74), with changes in variables and notation.)

We shall now specify the wave functions $\Psi$ of (7), (8) outside or on $\partial S^{4}$, in three steps: We define

$$
\zeta\left(r^{3}\right)= \begin{cases}+1, & \text { if } r^{3} \leq z_{a} \\ \text { undefined, } & \text { if } z_{a}<r^{3}<z_{b} \\ -1, & \text { if } r^{3} \geq z_{b}\end{cases}
$$

We also define

$$
X_{ \pm 1}\left(\mathbf{k}_{2} ; \mathbf{k}_{1}\right)=\theta\left( \pm k_{2}^{3}\right) \delta^{3}\left(\mathbf{k}_{2}-\mathbf{k}_{1}\right)+\theta\left(\mp k_{2}^{3}\right) S\left(\mathbf{k}_{2} ; \mathbf{k}_{1}\right) .
$$

where $\theta$ is the unit step function. The desired result is

$$
\Psi\left(t, \mathbf{r} ; \mathbf{k}_{1}\right)=\int d^{2} k_{2} \psi\left(t, \mathbf{r} ; \mathbf{k}_{2}\right) X_{\zeta\left(r^{3}\right)}\left(\mathbf{k}_{2} ; \mathbf{k}_{1}\right) .
$$

We note that (A9) entails the assumption that so long as $r^{3}$ is outside of $S^{4}$, but $t$ ventures inside $Q^{4}$, the properties of the $S$-matrix guarantee that the the time development of the wave functions in (A9) is still given correctly; conventionally, the $S$-matrix is developed in terms of its time dependence, as 
an input-to-output map valid only in mapping the pre-interaction to the postinteraction wave function, as in $(\mathrm{A} 6 \mathrm{~b})$. We are presuming that the same $S$ matrix also maps wave functions correctly when the input and output surfaces are merely exterior to $S^{4}$, whatever be the choice of time variable.

Let us define and calculate the $\mathbf{k}$-space matrix of the net probability flux $\mathcal{F}^{3}\left(R^{3}\right)$ flowing across planes $R^{3}=$ constant [compare (8)], for $R^{3} \leq z_{a}$ or for $R^{3} \geq z_{b}$. We find, respectively, that

$$
\begin{aligned}
\left\langle\Psi\left(\mathbf{k}_{2}\right)\right| & \mathcal{F}^{3}\left(R^{3}\right)\left|\Psi\left(\mathbf{k}_{1}\right)\right\rangle \\
= & \int d T d R^{1} d R^{2}\left\langle\Psi\left(\mathbf{k}_{2}\right)\left|\mathcal{J}^{3}\left(T, R^{1}, R^{2}, R^{3}\right)\right| \Psi\left(\mathbf{k}_{1}\right)\right\rangle \\
= & \zeta\left(R^{3}\right)\left[\theta\left(\zeta\left(R^{3}\right) k_{2}^{3}\right) \delta^{3}\left(\mathbf{k}_{2}-\mathbf{k}_{1}\right)\right. \\
& \left.-\int d^{3} k_{3} S^{\dagger}\left(\mathbf{k}_{2} ; \mathbf{k}_{3}\right) \theta\left(-\zeta\left(R^{3}\right) k_{3}^{3}\right) S\left(\mathbf{k}_{3} ; \mathbf{k}_{1}\right)\right]
\end{aligned}
$$

the equality of the two forms of (A10b) is equivalent to the unitarity of the $S$-matrix. In fact, because of (6), once the rhs of (A10b) is evaluated at an $R^{3} \leq z_{a}$, say, these values hold for any $R^{3}$, including $z_{a}<R^{3}<z_{b}$. When the $S$-matrix is block-diagonal (no reflections), the rhs of (A10b) reduces to (A3a). If the $S$-matrix is block-off-diagonal (no transmissions), the rhs of (A10b) is zero, corresponding to the fact that there is then zero net current for all $R^{3}$; hence, the combined spatial propagation operator $U^{(1)} \mathcal{J}^{3}$ zeroes wave functions in systems for which no transmissions occur, and is of little use in these cases. That the latter outcome is not associated with zero for the powers of the dwell time arises from the fact that in carrying out the integrals in (23) with the weight functions (24), it is not the net probability current but the net time current that is calculated - what results is a differential operator, as in (35).

We now exhibit how the $U^{(1)}$ operators of (A7) propagate the wave functions of (A9) along $z$, from $R^{3}$ to $r^{3}$ :

$$
\begin{aligned}
& \int d T d R^{1} d R^{2}\langle t\left., \mathbf{r}\left|U^{(1)} \mathcal{J}^{3}\left(T, R^{1}, R^{2}, R^{3}\right)\right| \Psi\left(\mathbf{k}_{1}\right)\right\rangle \\
&=\int d^{3} k_{2} \Psi\left(t, \mathbf{r} ; \mathbf{k}_{2}\right)\left\langle\Psi\left(\mathbf{k}_{2}\right)\left|\mathcal{F}^{3}\left(R^{3}\right)\right| \Psi\left(\mathbf{k}_{1}\right)\right\rangle
\end{aligned}
$$

The last result is independent of $R^{3}$.

\section{Appendix B: Lower bound for incommensurability}

We study the question of mean values and variances of system dynamical variables analogous to the dwell time, and of the compatibility or incommensurability of joint measurements of pairs of such dynamical variables, one of which can be the dwell time. "Joint measurements" does not mean simultaneous, as 
we are working primarily with spatial rather than temporal propagation, and the measurements determine flux correlations that occur at different times and spatial positions. In this connection, note that, in the Heisenberg picture, dynamical variables that commute at equal times generally do not commute when taken at unequal times. For example, the difference in $r^{1}$ positions of (D2) $(i)$, will normally not commute with the similarly defined difference in $r^{2}$ positions. In time intervals such that the interaction-free realm is attained by the particle both at input and at output, one might use the $S$-matrix to compute standard deviations and quantitative estimates of incompatibility for the measurement pairs; instead, we shall study measurements across $z$ intervals and the slab $S^{4}$.

Let $K$ and $L$ be Hermitean operators for which we want to compute mean and mean square differences of their values upon exit from, and entry to, the slab $S^{4}$; we shall also define an expectation value for a generalized commutator, and show that the product of the variances of $K$ and $L$ is greater than, or equal to, one-fourth of the square of the mean of the commutator.

Since the operators do not generally commute with $\mathcal{J}^{3}$, we use a symmetrized version to maintain the Hermitean property. We shall not construct a general current density for $K$ in the interior of $S^{4}$, but work with boundary fluxes only. The average net output minus input flux of $K$ for $S^{4}$ we call $K_{\partial S^{4} * * 1}$, which is taken to be

$$
\begin{aligned}
K_{\partial S^{4} * * 1}= & \int_{\partial S^{4}} d T d R^{1} d R^{2} \\
& \times\left.\operatorname{Tr}\left((1 / 2)\left(K \mathcal{J}^{3}(\Xi)+\mathcal{J}^{3}(\Xi) K\right) \rho^{(1)}\right)\right|_{R^{3}=z_{a}} ^{R^{3}=z_{b}} \\
= & \int d^{3} k_{1} \int d^{3} k_{2} \int_{\partial S^{4}} d T d R^{1} d R^{2} \\
& \times\left.\left\langle\Psi\left(\mathbf{k}_{1}\right)\left|(1 / 2)\left(K \mathcal{J}^{3}(\Xi)+\mathcal{J}^{3}(\Xi) K\right)\right| \Psi\left(\mathbf{k}_{2}\right)\right\rangle \tilde{\rho}\left(\mathbf{k}_{2} ; \mathbf{k}_{1}\right)\right|_{R^{3}=z_{a}} ^{R^{3}=z_{b}} .
\end{aligned}
$$

Similarly, we find for mean square of the flux difference $K_{\partial S^{4} * * 2}$

$$
\begin{aligned}
K_{\partial S^{4} * * 2}= & \int d^{3} k_{1} \int d^{3} k_{2} \int d^{3} k_{3} \int_{\partial S^{4}} d T_{1} d R_{1}^{1} d R_{1}^{2} \int_{\partial S^{4}} d T_{2} d R_{2}^{1} d R_{2}^{2} \\
& \times\left.\left\langle\Psi\left(\mathbf{k}_{3}\right)\left|(1 / 2)\left(K \mathcal{J}^{3}\left(\Xi_{1}\right)+\mathcal{J}^{3}\left(\Xi_{1}\right) K\right)\right| \Psi\left(\mathbf{k}_{1}\right)\right\rangle^{*}\right|_{R_{1}^{3}=z_{a}} ^{R_{1}^{3}=z_{b}} \\
& \times\left.\left\langle\Psi\left(\mathbf{k}_{3}\right)\left|(1 / 2)\left(K \mathcal{J}^{3}\left(\Xi_{2}\right)+\mathcal{J}^{3}\left(\Xi_{2}\right) K\right)\right| \Psi\left(\mathbf{k}_{2}\right)\right\rangle\right|_{2} ^{R_{2}^{3}=z_{b}} \\
& \times \tilde{\rho}\left(\mathbf{k}_{2} ; \mathbf{k}_{1}\right) .
\end{aligned}
$$

The variance $V(K)_{\partial S^{4}}$ and standard deviation $\Delta(K)_{\partial S^{4}}$ are calculated as in (30), (31), and (33), using the symmetrized operator product of $K$ and $\mathcal{J}^{3}$ instead of the time operator.

Next we follow, in part, a procedure given by Stoler and Newman [8] to obtain a quantative estimate of the incommensurability of measurements of $K$ and $L$. Let $\lambda$ be a real number, and define

$$
M(\lambda)=K+i \lambda L=M(-\lambda)^{\dagger}
$$


where the $\nmid$ means Hermitean conjugate in the spacetime sense. We take the variance of $M(\lambda)$ to be

$$
\begin{aligned}
V(M(\lambda))_{\partial S^{4}} & =\int d^{3} k_{1} \int d^{3} k_{2} \int d^{3} k_{3} \\
& \times\left[\int _ { \partial S ^ { 4 } } d T _ { 1 } d R _ { 1 } ^ { 1 } d R _ { 1 } ^ { 2 } \langle \Psi ( \mathbf { k } _ { 3 } ) | ( 1 / 2 ) \left(M(\lambda) \mathcal{J}^{3}\left(\Xi_{1}\right)\right.\right. \\
& \left.\left.+\mathcal{J}^{3}\left(\Xi_{1}\right) M(\lambda)\right)\left.\left|\Psi\left(\mathbf{k}_{1}\right)\right\rangle\right|_{R_{1}^{3}=z_{a}} ^{R_{3}^{3}=z_{b}}-\delta^{3}\left(\mathbf{k}_{3}-\mathbf{k}_{1}\right) M(\lambda)_{\partial S^{4} * * 1}\right]^{*} \\
& \times\left[\int _ { \partial S ^ { 4 } } d T _ { 2 } d R _ { 2 } ^ { 1 } d R _ { 2 } ^ { 2 } \left\langle\Psi \left(\mathbf{k}_{3} \mid(1 / 2)\left(M(\lambda) \mathcal{J}^{3}\left(\Xi_{2}\right)\right.\right.\right.\right. \\
& \left.\left.+\mathcal{J}^{3}\left(\Xi_{2}\right) M(\lambda)\right)\left|\Psi\left(\mathbf{k}_{2}\right\rangle\right|_{R_{2}^{3}=z_{a}}^{R_{a}^{3}=z_{b}}-\delta^{3}\left(\mathbf{k}_{3}-\mathbf{k}_{2}\right) M(\lambda)_{\partial S^{4} * * 1}\right] \\
& \times \tilde{\rho}\left(\mathbf{k}_{2} ; \mathbf{k}_{1}\right) .
\end{aligned}
$$

Since for every fixed $\mathbf{k}_{3}$ in the integrand, the remaining expression in (B4) is, in effect, the expectation value of a function in $\mathbf{k}$-space with respect to $\tilde{\rho}$, $V(M(\lambda))_{\partial S^{4}}$ is nonnegative. If we use (B3) in (B4), multiply out, and combine terms, we find that

$$
V(M(\lambda))_{\partial S^{4}}=V(K)_{\partial S^{4}}+\lambda^{2} V(L)_{\partial S^{4}}+\lambda C(K, L)_{\partial S^{4}},
$$

where $C(K, L)_{\partial S^{4}}$ is an expectation value of an entity that is plausibly described as a generalized commutator of $K$ and $L$, adapted to $\partial S^{4}$ :

$$
\begin{aligned}
C(K, L)_{\partial S^{4}} & =\frac{i}{4} \int d^{3} k_{1} \int d^{3} k_{2} \int d^{3} k_{3} \int_{\partial S^{4}} d T_{1} d R_{1}^{1} d R_{1}^{2} \int_{\partial S^{4}} d T_{2} d R_{2}^{1} d R_{2}^{2} \\
& \times\left\{\left[\left\langle\Psi\left(\mathbf{k}_{3}\right)\left|\left(K \mathcal{J}^{3}\left(\Xi_{2}\right)+\mathcal{J}^{3}\left(\Xi_{2}\right) K\right)\right| \Psi\left(\mathbf{k}_{2}\right)\right\rangle \tilde{\rho}\left(\mathbf{k}_{2} ; \mathbf{k}_{1}\right)\right.\right. \\
& \left.\times\left\langle\Psi\left(\mathbf{k}_{1}\right)\left|\left(L \mathcal{J}^{3}\left(\Xi_{1}\right)+\mathcal{J}^{3}\left(\Xi_{1}\right) L\right)\right| \Psi\left(\mathbf{k}_{3}\right)\right\rangle\right] \\
& -[K \leftrightarrows L]\}\left.\left.\right|_{R_{1}^{3}=z_{a}} ^{R_{1}^{3}=z_{b}}\right|_{R_{2}^{3}=z_{a}} ^{R_{2}^{3}=z_{b}}
\end{aligned}
$$

Note that $C(K, L)_{\partial S^{4}}$ is real. The quadratic expression in $\lambda$ of (B5) is nonnegative for all $\lambda$, so that the discriminant of the expression must be nonpositive, whereupon

$$
V(K)_{\partial S^{4}} V(L)_{\partial S^{4}} \geq(1 / 4)\left(C(K, L)_{\partial S^{4}}\right)^{2} ;
$$

(B7) is the desired inequality that sets a lower bound to the product of the variances of two output-minus-input fluxes on $\partial S^{4}$.

Something like a true uncertainty principle for arrival, or crossing, times of the particle at the $R^{3}=z_{b}$ plane might arise as follows: Choose $K$ and $L$ to be smoothly $R^{3}$-dependent in a way that they both take the value zero at $R^{3}=z_{a}$ and have a nonzero value at $R^{3}=z_{b}$, with zero $R^{3}$-derivatives at both $z_{a}$ and $z_{b}$. Then the values of (B1), (B2) and (B6) all have zero contributions from $R_{1,2}^{3}=z_{a}$. The problem then becomes that of choosing $K$, say, to be $T$ 
at $R^{3}=z_{b}$, and $L$ to be something like the conjugate momentum $p_{T}$ also at $R^{3}=z_{b}$, and trying to show that (B6) is nonzero for any physical $\tilde{\rho}$, including pure states. I have not attempted this line of investigation.

\section{Appendix C: Aharonov-Bohm operator}

In 1961, Aharonov and Bohm proposed an operator for the mean arrival time for a free particle at a specified (one dimensional) spatial position, given the quantum state at some initial time: see [9], [10], and for recent work, [11]. In this appendix, we state that what is in effect that operator can also be defined on single $z=$ constant slices of spacetime. Let $\tau(z)_{\tilde{\rho}}$ be the mean value of $T=$ time on the slice $R^{3}=z$ of spacetime. Then we have, omitting intermediate steps,

$$
\begin{aligned}
\tau(z)_{\tilde{\rho}} & =\int d^{3} k_{1} \int d^{3} k_{2} \int d T d R^{1} d R^{2} \\
& \times\left.\left\langle\psi\left(\mathbf{k}_{1}\right)\left|T \mathcal{J}^{3}\left(T, R^{1}, R^{2}, R^{3}\right)\right| \psi\left(\mathbf{k}_{2}\right)\right\rangle\right|^{R^{3}=z} \tilde{\rho}\left(\mathbf{k}_{2} ; \mathbf{k}_{1}\right) \\
& =\frac{m}{\hbar} \int d^{3} k_{1} \int d^{3} k_{2}\left\{\delta^{3}\left(\mathbf{k}_{1}-\mathbf{k}_{2}\right)\left[\frac{i}{2 k_{2}^{3}\left|k_{2}^{3}\right|}-\frac{i}{\left|k_{2}^{3}\right|} \frac{\partial}{\partial k_{2}^{3}}+\frac{z}{k_{2}^{3}}\right]\right. \\
& +\delta\left(k_{1}^{1}-k_{2}^{1}\right) \delta\left(k_{1}^{2}-k_{2}^{2}\right) \delta\left(k_{1}^{3}+k_{2}^{3}\right) \\
& \left.\times\left[-\frac{i}{2 k_{2}^{3}\left|k_{2}^{3}\right|} \exp \left(2 i k_{2}^{3} z\right)\right]\right\} \tilde{\rho}\left(\mathbf{k}_{2} ; \mathbf{k}_{1}\right) .
\end{aligned}
$$

In $(\mathrm{C} 1)$, the $\psi(\mathbf{k})$ 's are the free-particle wave functions of (A1). We remark that (35) is related to $(\mathrm{C} 2)$ by

$$
\tau\left(z_{b}\right)_{\tilde{\rho}}-\tau\left(z_{a}\right)_{\tilde{\rho}}=\left.\frac{m}{\hbar} \int d^{3} k_{1} \int d^{3} k_{2}\left\langle\Psi\left(\mathbf{k}_{1}\right)\left|\Lambda\left(z_{b}, z_{a}\right)\right| \Psi\left(\mathbf{k}_{2}\right)\right\rangle\right|^{\text {free }} \tilde{\rho}\left(\mathbf{k}_{2} ; \mathbf{k}_{1}\right) .
$$

\section{Appendix D: The basics of classical-to-quantum correlation functions}

In this section we shall attempt a physical motivation for the appearance of correlation functions in formulas for certain expectation values.

Conventional matrix elements of quantum operators specify values of the transition amplitude between two quantum states at a given instant of time. The mean dwell time for a particle in, say, the slab $S^{4}$ is a more delocalized entity that in effect involves integrals over a spacetime domain, or of the boundary of that domain - two disjoint, parallel three-planes in (16). Let us consider a familiar spacetime slab, call it $Q^{4}$, defined as follows:

$$
\begin{aligned}
Q^{4} & =\left\{(t, \mathbf{r}) \mid t_{a}<t<t_{b},-\infty<r^{1}, r^{2}, r^{3}<\infty\right\}, \\
\partial Q^{4} & =\left\{(t, \mathbf{r}) \mid t=t_{a} \text { or } t=t_{b},-\infty<r^{1}, r^{2}, r^{3}<\infty\right\} .
\end{aligned}
$$


Next, in classical dynamics we may want to address the following questions: Given an ensemble of classical dynamical trajectories $\left\{(\mathbf{r}(t), \mathbf{p}(t))\right.$, for $t_{a} \leq t \leq$ $\left.t_{b}\right\}$. We take $(\cdots)$ av to represent a classical ensemble average. Then $(i)$ what is the mean of the difference in $r^{1}$ at the final time and initial time? ( $\left.i i\right)$ what is the mean of the difference of the squares of $r^{1}$ at the initial and final time? (iii) what is the mean of the square of the difference of the final and initial $r^{1}$ ? and so on. For the ensemble we have the following averaged entities:

$$
\begin{aligned}
& \text { (i) }\left(r^{1}\left(t_{b}\right)\right)_{\mathrm{av}}-\left(r^{1}\left(t_{a}\right)\right)_{\mathrm{av}}, \\
& (\text { ii })\left(r^{1}\left(t_{b}\right)^{2}\right)_{\mathrm{av}}-\left(r^{1}\left(t_{a}\right)^{2}\right)_{\mathrm{av}}, \\
& \text { (iii) }\left(\left(r^{1}\left(t_{b}\right)-r^{1}\left(t_{a}\right)\right)^{2}\right)_{\mathrm{av}} .
\end{aligned}
$$

Let us rewrite $($ iii) as

$$
\text { (iii) } \begin{aligned}
\left(r^{1}\left(t_{b}\right)^{2}\right)_{\mathrm{av}} & +\left(r^{1}\left(t_{a}\right)^{2}\right)_{\mathrm{av}}-\left(r^{1}\left(t_{b}\right) r^{1}\left(t_{a}\right)\right)_{\mathrm{av}}-\left(r^{1}\left(t_{a}\right) r^{1}\left(t_{b}\right)\right)_{\mathrm{av}} \\
& =\left.\left.\left(r^{1}\left(t_{1}\right) r^{1}\left(t_{2}\right)\right)_{\mathrm{av}}\right|_{t_{1}=t_{b}} ^{t_{1}=t_{a}}\right|_{t_{2}=t_{a}} ^{t_{2}=t_{b}} .
\end{aligned}
$$

Moving to quantum mechanics, we need to hypothesize, what quantummechanical entity represents the classical correlation on the rhs of (D3)? If the Hamiltonian $H$ is time-independent, a plausible choice is (quantum averages with density matrix $\tilde{\rho}$ given at time $t=t_{a}$ are represented as $\left.(\cdots) \tilde{\rho}\right)$

$$
\text { (iii) }\left.\left.\left(r^{1}\left(t_{1}\right) r^{1}\left(t_{2}\right)\right)_{\tilde{\rho}}\right|_{t_{1}=t_{a}} ^{t_{1}=t_{b}}\right|_{t_{2}=t_{a}} ^{t_{2}=t_{b}},
$$

where the operators are

$$
\begin{aligned}
& r^{1}\left(t_{a}\right)=r^{1} \\
& r^{1}\left(t_{b}\right)=\exp \left(i H\left(t_{b}-t_{a}\right) / \hbar\right) r^{1} \exp \left(-i H\left(t_{b}-t_{a}\right) / \hbar\right) .
\end{aligned}
$$

We can also infer that the classical expression

$$
\text { (iv) }\left(\left(r^{1}\left(t_{b}\right)-r^{1}\left(t_{a}\right)\right)^{N}\right)_{\mathrm{av}}
$$

plausibly upgrades into the quantum-mechanical expression

$$
\text { (iv) }\left.\left.\left.\left(r^{1}\left(t_{1}\right) r^{1}\left(t_{2}\right) \cdots r^{1}\left(t_{N}\right)\right)_{\tilde{\rho}}\right|_{t_{1}=t_{a}} ^{t_{1}=t_{b}}\right|_{t_{2}=t_{a}} ^{t_{2}=t_{b}} \cdots\right|_{t_{N}=t_{a}} ^{t_{N}=t_{b}} .
$$

Skipping some steps, we can infer for time-dependent Hamiltonians that (iii) becomes

$$
\begin{aligned}
\int_{\partial Q^{4}} d^{3} R_{1} & \int_{\partial Q^{4}} d^{3} R_{2}\left[R_{1}^{1} R_{2}^{1}\right. \\
& \left.\times\left(J^{0_{1} 0_{2}}\left(T_{1}, \mathbf{R}_{1} ; T_{2}, \mathbf{R}_{2}\right)\right)_{\tilde{\rho}}\right]\left.\left.\right|_{T_{1}=t_{a}} ^{T_{1}=t_{b}}\right|_{T_{2}=t_{a}} ^{T_{2}=t_{b}} \\
& =\int_{Q^{4}} d^{4} \Xi_{1} \int_{Q^{4}} d^{4} \Xi_{2}\left(J^{1_{1} 1_{2}}\left(\Xi_{1} ; \Xi_{2}\right)\right)_{\tilde{\rho}}
\end{aligned}
$$


where the last step follows from Gauss's theorem.

The lhs of (D8) simplifies when $T_{1}=T_{2}=t_{b}$, say. Since according to (8) and (5) the operator $U^{(1)} \mathcal{J}^{0}$, when integrated over a $T=$ constant slice of spacetime, reduces to the unit operator at equal times, this contribution simplifies to a single-flux computation:

$$
\int d^{3} R_{1}\left(R_{1}^{1}\right)^{2}\left(J^{0_{1}}\left(t_{b}, \mathbf{R}_{1}\right)\right)_{\tilde{\rho}} .
$$

\section{References}

[1] E. Pollak and W. H. Miller, Phys. Rev. Lett. 53, 115 (1984)

[2] J. Muñoz, D. Seidel, and J. G. Muga, Phys. Rev. A 79, 012108 (2009)

[3] L. I. Schiff, Quantum Mechanics, McGraw-Hill, New York, NY, USA (1968), 3rd edition

[4] L. E. Ballentine, Quantum Mechanics, World Scientific, Singapore (1998).

[5] G. E. Hahne, Phys. Rev. A 80, 062101 (2009).

[6] G. E. Hahne, unpublished work.

[7] G. E. Hahne, J. Phys. A 36, 7149 (2003). An improved version (two references were added, but the equation numbers are unchanged) is in arXiv:quant-ph/0404012.

[8] D. Stoler and S. Newman, Phys. Lett. A 38, 433 (1972).

[9] Y. Aharonov and D. Bohm, Phys. Rev. 122, 1649 (1961)

[10] Y. Aharonov and D. Bohm, Phys. Rev. 134, B1417 (1964)

[11] I. L. Egusquiza, J. G. Muga, and A. D. Baute, in Time in Quantum Mechanics edited by J. G. Muga, R. S. Mayato, and I. L. Egusquiza, Springer, Berlin (2008), 2nd edition, Vol. 1, Ch. 10. 